\title{
O conhecimento dos fisioterapeutas sobre cuidados paliativos em pediatria em um hospital materno infantil
}

\author{
The knowledge of physical therapists on \\ palliative care in pediatria em a maternal \\ children"s
}

\section{Joice Lilian Rios de Oliveira' ${ }^{1}$ Roquenei da Purificação Rodrigues ${ }^{2}$ (1) Ludimille Azevedo Barreto ${ }^{3}$ (1)}

'Autora para correspondência. Faculdade Adventista da Bahia (Salvador). Bahia, Brasil. joiceh-lilian96@hotmail.com ${ }^{2}$ Hospital Estadual da Criança (Feira de Santana). Bahia, Brasil. roquenei@gmail.com ${ }^{3}$ Faculdade Adventista da Bahia (Salvador). Bahia, Brasil. ludimille.barreto@gmail.com

\begin{abstract}
RESUMO | INTRODUÇÃO: A falta de manejo em lidar com as crianças no período de terminalidade da vida pode tornar-se uma barreira entre o profissional e a família. Estudos sobre o entendimento dos profissionais de saúde, especialmente os fisioterapeutas a respeito da paliação na pediatria, são significativos para estabelecer estratégias de para conforto. OBJETIVO: descrever o conhecimento dos fisioterapeutas sobre a paliação na pediatria. MATÉRIAS E MÉTODOS: estudo quantitativo, descritivo, transversal, onde foi aplicado a adaptação do questionário pré-estruturado: Bonn Palliative Care Knowledge Test-BPW e um questionário sócio profissional, nos fisioterapeutas do Hospital materno infantil. RESULTADOS: A amostra foi constituída de 44 fisioterapeutas, de ambos os sexos, com idade variando entre 20-50 anos, com tempo de formação entre 1-15 anos. A análise do percentual de acertos no questionário de conhecimento no BPW, no que concerne ao item da filosofia dos CP 27,3\% responderam corretamente, já em relação a prioridade do tratamento médico em CP 38,7\% acertaram a resposta. CONCLUSÃO: $O$ entendimento sobre os CP ainda é superficial, a maioria das instituições de ensino está formando profissionais carentes conhecimento sobre as premissas do CP.
\end{abstract}

PALAVRAS-CHAVE: Cuidados paliativos. Pediatria. Fisioterapeutas.

\begin{abstract}
INTRODUCTION: The lack of management in dealing with children in the terminal period of life can become a barrier between the professional and the family. Studies on the understanding of health professionals, especially physiotherapists regarding palliation in pediatrics, are meaningful to establish comfort strategies. OBJECTIVE: to describe the knowledge of physiotherapists on palliation in pediatrics. MATERIALS AND METHODS: quantitative, descriptive, cross-sectional study, where the adaptation of the pre-structured questionnaire was applied: Bonn Palliative Care Knowledge Test-BPW and a socioprofessional questionnaire, in the physiotherapists of the maternal children's hospital. RESULTS: The sample consisted of 44 physiotherapists, of both sexes, with ages varying between 20-50 years, with training time between 1-15 years. The analysis of the percentage of correct answers in the knowledge questionnaire in the BPW, with regard to the item of the philosophy of the CP $27.3 \%$ answered correctly, in relation to the priority of the medical treatment in CP 38.7\% correct the answer. CONCLUSION: The understanding about PC is still superficial, most educational institutions are training professionals in need of knowledge about the premises of PC.
\end{abstract}

KEYWORDS: Palliative care. Pediatrics. Physiotherapists. 


\section{Introdução}

O avançar tecnológico propiciou a sobrevida de crianças consideradas graves, concomitantemente desenvolveu-se um grupo de crianças com doenças crônicas e dependentes de tecnologia assistiva, muitas vezes com expectativa de vida reduzida e que acabam necessitando de repetidas internações hospitalares. ${ }^{1}$

Lidar com esse novo perfil de paciente exige dos profissionais de saúde uma abordagem diferente, visando à integralidade dos aspectos psicológicos e espirituais no cuidado ao paciente surgindo assim os Cuidados Paliativos (CP).. , 3

A Organização Mundial da Saúde (OMS), em 2002, definiu Cuidado Paliativo como "uma abordagem que melhora a qualidade de vida de pacientes (adultos e crianças) e seus familiares, que enfrentam doenças que ameacem a vida", prevenindo e aliviando o sofrimento através da identificação precoce, avaliação correta e tratamento da dor e outros problemas físicos, psíquicos, sócio familiares e espirituais. 4

Cerca de $98 \%$ das crianças que necessitam de cuidados paliativos residem em países subdesenvolvidos como o Brasil. Menos de $10 \%$ dos hospitais no Brasil possuem equipe de cuidados paliativos, entretanto o número de pacientes que necessitam de paliação e conseguem obter é bem inferior. 5,6

Um dos princípios básicos para o cuidado paliativo infantil é o cuidado focado na criança, orientado a toda família, construído com uma boa relação equipe-família. Para tanto, a equipe multiprofissional deve estar apta a atender as necessidades da criança de forma integral, viabilizando ações que garantam uma sobrevida digna e controle adequado dos sintomas compreendendo o paciente e sua família na sua subjetividade e complexidade. 7,8

A falta de manejo em cuidar das crianças no período de terminalidade da vida pode se tornar uma barreira entre o profissional e a família. Por conseguinte, faz-se necessário que o profissional compreenda como proceder devidamente durante a paliação. O fisioterapeuta como membro da equipe multidisciplinar deve basear sua intervenção nas especificidades da criança, compreendendo em sua total integralidade. 5,6
A discussão sobre cuidados paliativos na pediatria e sobre o conhecimento que os profissionais de saúde, enfatizando os fisioterapeutas, possuem sobre os mesmos, ainda é recente no Brasil e por isso acredita-se que a temática esteja em processo de construção. Estudos sobre o entendimento dos profissionais de saúde, de um modo particular os fisioterapeutas a respeito da paliação na pediatria, são significativos para estabelecer estratégias de sensibilização, com o intuito de buscar assistência humanizada, de forma que o conforto da criança seja priorizado..-7

Nesse contexto, o presente estudo objetivou descrever o conhecimento dos fisioterapeutas sobre a paliação na pediatria.

\section{Material e métodos}

Trata-se de um estudo quantitativo, de natureza descritiva, com desenho de estudo transversal, onde foram aplicados dois questionarios nos fisioterapeutas de Hospital materno infantil na cidade de Feira de Santana, Bahia-Brasil. A pesquisa iniciou se após aprovação da Comissão de Pesquisa e Ética do Hospital Irmã Dulce e da Comisão de Ética e Pesquisa da instituição onde a pesquisa foi realizada (Parecer n. ${ }^{\circ} 4.304 .730$, de 28 de setembro de 2020 ).

Foram convidados a participar do estudo os fisioterapeutas de um hospital materno infantil. Os mesmos são atuantes nas Unidades de Terapia Intensiva Pediátrica e Neonatal, e/ou nos setores da Emergência, Uncino, Ucinca Enfermaria, devido a rotatividade dos profissionais dentre esses setores, e o fisioterapeutas lideres setoriais em decorrência da sua participação ativa dentro das unidades. Não foram incluídos os profissionais que atuam na assistência obstétrica, que estiverem em cargos de coordenação, com licença por motivos de saúde ou maternidade, férias no período da coleta e os pesquisadores deste estudo.

Inicialmente foi realizado a aplicação de um questionário sócio profissional composto por 15 questões fechadas (marca um $\mathrm{x}$ ). $\mathrm{O}$ questionário possui perguntas referentes ao sexo, idade, área de atuação, tempo de formação e de atuação, existência de formação complementar e questões tocantes ao processo de morte e morrer. 
Posteriormente os profissionais responderam uma versão em português, adaptada transculturalmente pelos pesquisadores do Bonn Palliative Care Knowledge Test (BPW), do qual foram selecionadas as questões inerentes a população alvo desse estudo. O BPW é um teste auto aplicável, anônimo e com o tempo estimado para resposta de 10 minutos. Neste estudo, o BPW continha 15 questões para a análise de conhecimentos gerais sobre cuidados paliativos e sobre o processo de morte e morrer.

A escala contida no questionário é do tipo Likert, na qual os respondentes marcaram a validade dos itens seguindo os termos "correto", "razoavelmente correto", "pouco correto" ou "incorreto", como na descrição de classificação abaixo.

Cada afirmativa dos tópicos de análise de conhecimento possui duas respostas esperadas "correto" e "razoavelmente correto" ou "pouco correto" e "incorreto". Dessa forma, as questões 1,3,4,5,6,8,10,12,13,14, foram consideradas respondidas corretamente quando apenas uma alternativa foi marcada como "pouco correto" ou "incorreto"; já os itens 2,7,9,11,15, as respostas esperadas eram "correto" ou "razoavelmente correto".9

Os itens do questionário com a frequência de respostas mais discrepantes, serão analisados individualmente, onde a respostas serão divididas em corretas e erradas, de acordo com o referencial do questionário, sendo as mesmas quantificadas e expostas graficamente.

As variáveis categóricas foram apresentadas em contagens de frequência e porcentagens numéricas. As análises foram realizadas no programa IBM SPSS Statistics Versão 26.

\section{Resultados}

A amostra foi constituída de 44 fisioterapeutas, de ambos os sexos, com idade variando entre 20-50 anos Tabela 1.

Tabela 1. Perfil social dos fisioterapeutas que trabalham com neonatologia e pediatria-2020

\begin{tabular}{l|l}
\hline Sexo & n $(\%)$ \\
\hline Feminino & $35(79,5)$ \\
Masculino & $9(20,5)$ \\
& \\
\hline Idade & $17(38,6)$ \\
\hline $20-30$ anos & $24(54,5)$ \\
$31-40$ anos & $3(6,8)$ \\
\hline $41-50$ anos & \\
\hline Elaborado pelos autores,2021 & \\
Fonte: Pesquisa de campo, 2020 &
\end{tabular}

No questionário sócioprofissional os participantes responderam qual tempo de formado, tempo de trabalho na área de Neonatologia/Pediatria e responderam "sim" ou "não" para perguntas sobre a execução de cursos sobre cuidados paliativos e sobre acreditar que o conteúdo que aprendeu durante a faculdade foi suficiente para lidar com paciente em situação terminal Tabela 2.

Com relação ao tempo de ocupação profissional, 61,4\% dos fisioterapeutas possuem de 1 a 5 anos de atuação Tabela 2. No que concerne ao tempo de trabalho na área de Neonatologia e Pediatria, 56,8\% tem de 1 a 5 anos a Tabela 2.

Ao abordar sobre informações sobre o cuidado do paciente terminal durante a graduação, 93,2\% responderam que não tiveram Tabela 2. No tocante a formação complementar com a temática cuidados paliativos, 65,9\% nunca realizaram tal formação, depois de formado Tabela 2. 
Tabela 2. Perfil profissional dos fisioterapeutas que trabalham com neonatologia e pediatria-2020

\begin{tabular}{l|c}
\hline Tempo de Formação & N $(\%)$ \\
\hline$<1$ ano & $1(2,3)$ \\
$1-5$ anos & $27(61,4)$ \\
$6-10$ anos & $3(6,8)$ \\
$11-15$ anos & $2(4,5)$ \\
$16-20$ anos & $11(25)$ \\
\hline Tempo de atuação na área & \\
\hline$<1$ ano & $1(2,3)$ \\
$1-5$ anos & $25(56,8)$ \\
$6-10$ anos & $2(4,5)$ \\
$11-15$ anos & $11(29,5)$ \\
\hline Informações sobre cuidado do paciente & \\
terminal durante a graduação & $3(6,8)$ \\
\hline Sim & $41(93,2)$ \\
Não & $15(34,1)$ \\
\hline Realizou curso de cuidados paliativos & $29(65,9)$ \\
\hline Sim & \\
Não & \\
\hline Elaborado pelos autores,2021 & \\
Fonte: Pesquisa de campo, 2020
\end{tabular}

A análise do desempenho geral no BPW considerou todos os questionários onde os participantes, responderam apenas uma opção em cada resposta. Todos os participantes responderam corretamente o questionário totalizando o total de 44 questionários. As respostas obtidas seguem explanadas na tabela abaixo.

Os resultados da avaliação de conhecimentos por meio do Bonn Palliative Care Knowledge Test encontram- se na Tabela 3.

Tabela 3. Análise dos itens da secção conhecimento do questionário BPW adaptado-2020 (continua)

\begin{tabular}{|c|c|}
\hline $\begin{array}{l}\text { 1.Os CP nunca devem ser combinados com } \\
\text { tratamentos curativo }\end{array}$ & $\mathrm{N}(\%)$ \\
\hline $\begin{array}{l}\text { Correto } \\
\text { Razoavelmente Correto } \\
\text { Pouco Correto } \\
\text { Incorreto }\end{array}$ & $\begin{array}{r}7(15,9) \\
11(25,0) \\
8(18,2) \\
18(40,9)\end{array}$ \\
\hline \multicolumn{2}{|c|}{$\begin{array}{l}\text { 2. As terapias não farmacológicas (por exemplo, } \\
\text { fisioterapia) são importantes na gestão da dor }\end{array}$} \\
\hline $\begin{array}{l}\text { Correto } \\
\text { Razoavelmente Correto } \\
\text { Pouco Correto }\end{array}$ & $\begin{array}{c}40(90,5) \\
3(6,8) \\
1(2,3)\end{array}$ \\
\hline \multicolumn{2}{|l|}{$\begin{array}{l}\text { 3.Para os familiares é sempre importante permanecer } \\
\text { junto à pessoa nas últimas horas de vidaaté que a } \\
\text { morte ocorra }\end{array}$} \\
\hline $\begin{array}{l}\text { Correto } \\
\text { Razoavelmente Correto } \\
\text { Pouco Correto }\end{array}$ & $\begin{array}{c}33(75,0) \\
10(22,7) \\
1(2,3)\end{array}$ \\
\hline \multicolumn{2}{|l|}{$\begin{array}{l}\text { 4.Os CP requerem uma proximidade emocional } \\
\text { constante }\end{array}$} \\
\hline $\begin{array}{l}\text { Correto } \\
\text { Razoavelmente Correto } \\
\text { Pouco Correto } \\
\text { Incorreto }\end{array}$ & $\begin{array}{c}32(72,7) \\
10(22,7) \\
1(2,3) \\
1(2,3)\end{array}$ \\
\hline \multicolumn{2}{|l|}{$\begin{array}{l}\text { 5.Como avanço da idade,as pessoas aprenderam alidar } \\
\text { com a dor de forma independente,em resultado } \\
\text { devária sexperiências }\end{array}$} \\
\hline $\begin{array}{l}\text { Correto } \\
\text { Razoavelmente Correto } \\
\text { Pouco Correto } \\
\text { Incorreto }\end{array}$ & $\begin{array}{c}5(11,4) \\
16(36,4) \\
10(22,7) \\
13(29,5)\end{array}$ \\
\hline
\end{tabular}




\begin{tabular}{|c|c|}
\hline $\begin{array}{l}\text { 6.A filosofia dos CP preconiza que não sejam } \\
\text { realizadas quaisquer intervenções destinadas a } \\
\text { prolongar a vida }\end{array}$ & \\
\hline $\begin{array}{l}\text { Correto } \\
\text { Razoavelmente Correto } \\
\text { Pouco Correto } \\
\text { Incorreto }\end{array}$ & $\begin{array}{l}18(40,9) \\
14(31,8) \\
3(6,8) \\
9(20,5)\end{array}$ \\
\hline 7.O limiar da dor é diminuído pela ansiedade ou fadiga & \\
\hline $\begin{array}{l}\text { Correto } \\
\text { Razoavelmente Correto } \\
\text { Pouco Correto } \\
\text { Incorreto }\end{array}$ & $\begin{array}{l}7(15,9) \\
2(4,5) \\
14(31,8) \\
21(47,8)\end{array}$ \\
\hline $\begin{array}{l}8 \text { As pessoas com doenças que ameaçam a vida devem } \\
\text { ser sempre informadas da verdade, para que possam } \\
\text { preparar o seu processo de morrer }\end{array}$ & \\
\hline $\begin{array}{l}\text { Correto } \\
\text { Razoavelmente Correto }\end{array}$ & $\begin{array}{l}34(77,3) \\
10(22,7)\end{array}$ \\
\hline $\begin{array}{l}\text { 9.0s membros da equipe não têm de ser crentes para } \\
\text { prestar cuidados espirituais à pessoa em fim de vida. }\end{array}$ & \\
\hline $\begin{array}{l}\text { Correto } \\
\text { Razoavelmente Correto } \\
\text { Pouco Correto } \\
\text { Incorreto }\end{array}$ & $\begin{array}{l}26(59,1) \\
11(25) \\
3(6,8) \\
4(9,1)\end{array}$ \\
\hline 10.A pessoa que recebe $C P$ deve aceitar amorte & \\
\hline $\begin{array}{l}\text { Correto } \\
\text { Razoavelmente Correto } \\
\text { Pouco Correto } \\
\text { Incorreto }\end{array}$ & $\begin{array}{l}2(4,5) \\
14(31,8) \\
15(34,1) \\
13(29,5)\end{array}$ \\
\hline $\begin{array}{l}\text { 11.As competências de comunicação podem ser } \\
\text { aprendidas }\end{array}$ & \\
\hline $\begin{array}{l}\text { Correto } \\
\text { Razoavelmente Correto }\end{array}$ & $\begin{array}{c}35(79,5) \\
9(20,5)\end{array}$ \\
\hline $\begin{array}{l}\text { 12.Os outros pacientes não devem ser informados } \\
\text { sobre amorte da pessoa para evitar inquietações }\end{array}$ & \\
\hline $\begin{array}{l}\text { Correto } \\
\text { Razoavelmente Correto } \\
\text { Pouco Correto } \\
\text { Incorreto }\end{array}$ & $\begin{array}{c}8(18,2) \\
20(45,5) \\
11(25,0) \\
5(11,4)\end{array}$ \\
\hline $\begin{array}{l}13.0 \text { tratamento médico tem sempre prioridade nos } \\
\text { CP }\end{array}$ & \\
\hline $\begin{array}{l}\text { Correto } \\
\text { Razoavelmente Correto } \\
\text { Pouco Correto } \\
\text { Incorreto }\end{array}$ & $\begin{array}{c}13(29,5) \\
14(31,8) \\
6(13,6) \\
11(25)\end{array}$ \\
\hline $\begin{array}{l}\text { 14.Quando morre uma pessoa,os rituais visíveis e as } \\
\text { cerimónias de despedidas devem ser evitadas para } \\
\text { não causar inquietações }\end{array}$ & \\
\hline $\begin{array}{l}\text { Correto } \\
\text { Razoavelmente Correto } \\
\text { Pouco Correto } \\
\text { Incorreto }\end{array}$ & $\begin{array}{l}3(6,8) \\
5(11,4) \\
7(15,9) \\
29(65,9)\end{array}$ \\
\hline 15. A fase final refere-se aosúltimos 3 dias de vida & \\
\hline $\begin{array}{l}\text { Correto } \\
\text { Razoavelmente Correto } \\
\text { Pouco Correto } \\
\text { Incorreto }\end{array}$ & $\begin{array}{l}4(9,1) \\
8(18,2) \\
11(25,0) \\
21(47,7)\end{array}$ \\
\hline
\end{tabular}

Elaborada pelos autores, 2021

Fonte:Pesquisa de campo,2020 
Posteriormente a avaliação geral, os itens dos questionários foram analisados individualmente, sendo mensurado o quantitativo de respostas corretas e erradas.

A questões 6 e 13 representaram os itens que obtiveram maior discrepância nas respostas, sendo as mesmas respondidas erroneamente com maior repetição (Figura 1 e Figura 2). Dos participantes, 72,7\% acreditam que a filosofia dos cuidados paliativos não prioriza que sejam realizadas intervenções destinadas a prolongar a vida dos pacientes em cuidados paliativos Figura 1.

Figura 1. Porcentagem de resposta certa e errada da afirmativa: “A filosofia dos CP preconiza que não sejam realizadas quaisquer intervenções destinadas a prolongar a vida"

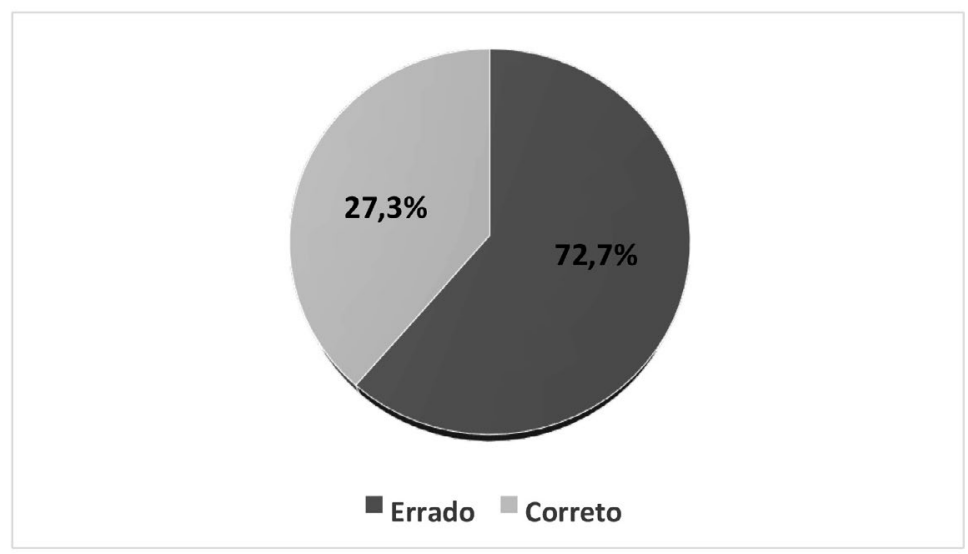

Elaborado pelos autores, 2021

Fonte: Pesquisa de campo, 2020

Com relação ao item que relata que o tratamento médico sempre tem prioridade nos cuidados paliativos $61,3 \%$ responderam de maneira errônea Figura 2.

Figura 2. Porcentagem de resposta certa e errada da afirmativa: $\mathrm{O}$ tratamento médico tem sempre prioridade nos $\mathrm{CP}$

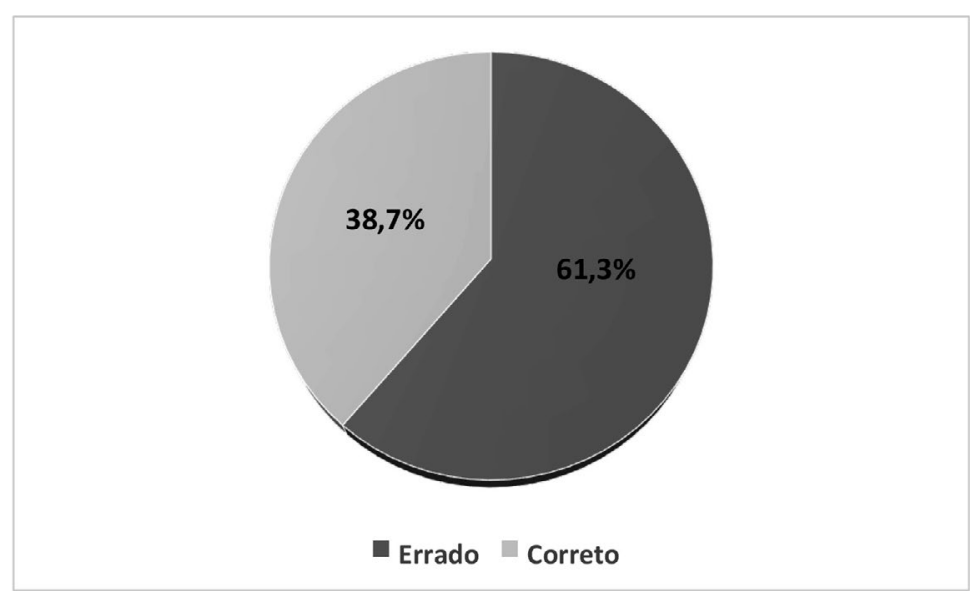

Elaborado pelos autores, 2021

Fonte: Pesquisa de campo, 2020 


\section{Discussão}

O desconhecimento dos profissionais é um fator importante para que os pacientes não tenham acesso a medidas paliativistas, estudos e debates sobre o tema são importantes para a atualização técnico-científica dos profissionais. Pesquisas realizadas em outros países expuseram que existe um déficit na formação dos profissionais de saúde no que concerne os cuidados paliativo. ${ }^{10}$ Cerca de $93,2 \%$ dos participantes deste estudo relataram que não obtiveram informações sobre o cuidado do paciente terminal durante a gradução. Analógo ao objetivo deste estudo, Oliveira, Teixeira, Tavares (2019) utilizaram o BPW para verificar o nível de conhecimento dos profissionais dentro de um CTI (Centro de Terapia Intensiva), expondo ao final da pesquisa que o reflexo da baixa oferta de cursos na área dos CP durante a graduação, reflete dentro do CTI estudado onde apenas $22 \%$ dos participantes possuíam formação específica em $\mathrm{CP} \frac{12}{2}$, semelhante ao resultado desta pesquisa onde $34,1 \%$ do fisioterapeutas relataram possuir algum curso em CP.

A fisioterapia ainda possui sua formação baseada no modelo biomédico, onde se prediz a separação entre corpo e mente e a inexistência da importância da espiritualidade no processo saúde doença. A grade curricular com seu enfoque reabilitador ainda centraliza suas disciplinas na busca da reabilitação de doenças que restringem a locomoção humana. ${ }^{11}$

A criação de disciplinas optativas é uma opção para suplantar esse atraso, entretanto ainda se faz necessário despertar o interesse dos graduandos sobre o tema, pois o mesmo pode ser levado a vida profissional, cerca de $65,9 \%$ dos profissionais nunca realizaram curso de cuidados paliativos, consolidando informação anterior..$^{11}$

Em dezembro de 2010, o Ministério da Saúde, através da Portaria $n^{\circ} 4.279$, estabeleceu os cuidados paliativos nas suas diretrizes de atenção à saúde, entretanto evidente a desinformação sobre os princípios que norteiam essa modalidade de assistência. ${ }^{13}$

Contudo, os CP devem ser iniciados desde o diagnóstico e as medidas curativas e devem ser usados desde que proporcionem qualidade de vida aos pacientes, conforme os princípios da OMS. Dessa forma os pacientes recebem $\mathrm{CP}$ em momentos diferentes da evolução da doença não os privando, do tratamento médico associado a uma abordagem que inclui espiritualidade entre as dimensões do ser humano., 5, , ,15,16

Neste estudo, os itens do BPW que afirmam que "Os $\mathrm{CP}$ nunca devem ser combinados com tratamentos curativos" e que "A filosofia dos CP preconiza que não sejam realizadas quaisquer intervenções destinadas a prolongar a vida" foram julgados de forma correta por 15,9 e 40,9 respectivamente dos participantes.

Um dos princípios do CP é a integralidade do cuidado, auxiliar o paciente e sua família no enfretamento e alivio dos sintomas físicos e psicológicos. ${ }^{6,7}$ A dor é considerada um dos sintomas mais incapacitantes na criança, o item 2 do BPW refere sobre a importância das terapias não farmacológicas são importantes na gestão da dor 90,5\% acertaram a questão. Na adaptação transcultural do BPW realizada por Minosso, Martins, Oliveira, $80 \%$ dos profissionais e dos estudantes que participaram da pesquisa responderam este item corretamente. ${ }^{9}$

O manual dos CP refere que o sucesso do tratamento para gestão da dor deve ser elaborado pela equipe interdisciplinar onde a fisioterapia deve atuar por meio de técnicas e exercícios buscando a confortabilidade da criança.,$\underline{, 6}$

Ainda no contexto de cuidado integral, a dimensão espiritual é de grande importância para os pacientes e seus familiares. A abordagem espiritual quando se lida com sofrimento e dor possui efeito positivo frente aos desafios da vida promovendo bem estar. ${ }^{17} \mathrm{O}$ manual da ANCP traz que os membros da equipe não têm que ser crentes para abordar o paciente sob o ponto de vista da espiritualidade, o que se confunde e se sobrepõem invariavelmente a questão religiosa..$^{5,6}$

A espiritualidade é um aspecto de transcendência do significado da vida aliado ou não a religião, 59,1\% dos participantes deste estudo consideraram correto o item referente à religiosidade e cuidados espirituais prestados pelos membros da equipe. Para $66,7 \%$ dos estudantes e $80 \%$ dos profissionais participantes do trabalho de Minosso, Martins, Oliveira, o item citado anteriormente é considerado correto. ${ }^{9}$

Spineli transcreveu que para os enfermeiros que atuam nas APS (Atenção Primaria a Saúde) a espiritualidade relaciona-se a questão de fé e religiosidade sendo uma abordagem da dimensão espiritual e da existência..$^{18}$ 
A construção do vínculo entre a equipe e o paciente, transcorre através de uma comunicação efetiva. Comunicar-se adequadamente possibilita a troca e compreensão de ideias, pensamentos e propósitos..$^{19}$

Para saber falar e escutar ativamente pacientes e familiares, estratégias de comunicação interpessoal podem e devem ser aprendidas. . $^{20}$ Neste estudo, o item 11, referente à aprendizagem de comunicação, apresentou $100 \%$ de resposta correta. Este ítem na pesquisa de Pfister et al. obteve $76,2 \%$ de acertos por parte dos participantes e $100 \%$ pelos especialistas. $\underline{\underline{22}}$

Spineli expôs que os participantes da sua pesquisa discorreram, que na abordagem paliativista a comunicação relaciona-se principalmente à necessidade de informação dos pacientes e familiares a comunicação de noticiais sendo considerada um ponto fundamental. Este item apresentou maior percentual de acertos $96,9 \%$ resultado que evidencia que de acordo com os profissionais a comunicação em CP podem ser aprendidas. $\frac{18}{}$ Neste sentido, a comunicação constitui um dos pilares para o cuidado efetivo, possibilitando que a equipe direcione seu atendimento de uma forma coesa com cuidados direcionados para a individualidade do paciente, interação família equipe clara e objetiva, o que implica em uma melhor assistência.

Portanto, existe a necessidade de mais publicações e debates sobre o assunto. O BPW traduzido e adaptado para o português possibilita o conhecimento dos principais déficits e medidas de sensibilização e educação continuada perante a equipe analisada.

Os resultados deste estudo podem ter limitações relacionadas à aplicação do questionário, por se tratar de um questionário autoaplicável, além de ter sido respondido durante o horário de trabalho dos participantes, podendo limitar a atenção e o tempo dedicado ao seu preenchimento.

\section{Conclusão}

O presente estudo corrobora com a realidade de que o entendimento sobre os cuidados paliativos ainda é superficial, a maioria das instituições de ensino está formando profissionais carentes de conhecimento sobre as premissas dos Cuidados Paliativos.

\section{Contribuições dos autores}

Oliveira JLR e Purificação R Rodrigues e Barreto LA participaram da elaboração do desenho de estudo, coleta de dados, análise e interpretação dos resultados e redação do manuscrito.

\section{Conflitos de interesses}

Nenhum conflito financeiro, legal ou político envolvendo terceiros (governo, empresas e fundações privadas, etc.) foi declarado para nenhum aspecto do trabalho submetido (incluindo, mas não se limitando a subvenções e financiamentos, participação em conselho consultivo, desenho de estudo, preparação de manuscrito, análise estatística, etc.).

\section{Referências}

1. Ministério da Saúde (Brasil), Instituto Nacional de Câncer José Alencar Gomes da Silva, Instituo Ronald Macdonald. Diagnóstico Precoce do Câncer na Criança e no Adolescente [Internet]. $2^{\mathrm{a}}$. ed. Rio de Janeiro: INCA; 2013. Disponível em: http://bvsms.saude. gov.br/bvs/publicacoes/diagnostico_precoce_cancer_crianca_2ed. pdf

2. Piva P, Garcia P, Lago P. Dilemas e dificuldades envolvendo decisões de final de vida e oferta de cuidados paliativos em pediatria. Rev. bras. ter. intensiva. 2011;23(1):78-86. https://doi. org/10.1590/S0103-507X2011000100013

3. Melo A. Os Cuidados Paliativos no Brasil. In: Pessini L, Bertachini L, organizadores. Humanização e Cuidados Paliativos. São Paulo: Edições Loyola; 2011.

4. World Health Organization. Cancer Pain Relief and Palliative Care in Children [Internet]. Geneva: WHO; 1998. Disponível em: http://whqlibdoc.who.int/publications/9241545127.pdf

5. Academia Nacional de Cuidados Paliativos. Manual de Cuidados Paliativos ANCP [Internet]. $2^{\text {a }}$. ed. São Paulo: ANCP; 2012. Disponível em: http://biblioteca.cofen.gov.br/wp-content/ uploads/2017/05/Manual-de-cuidados-paliativos-ANCP.pdf

6. Academia Nacional de Cuidados Paliativos (ANCP). Análise Situacional e Recomendações para Estruturação de Programas de Cuidados Paliativos no Brasil. São Paulo: Academia Nacional de Cuidados Paliativos; 2018.

7. Bispo Júnior JP. Fisioterapia e saúde coletiva: desafios e novas responsabilidades profissionais. Ciênc. saúde coletiva. 2010;15(supl 1):1627-36. https://doi.org/10.1590/S1413$\underline{81232010000700074}$

8. Lago PM, Devictor D, Piva JP, Bergounioux J. Cuidados de Final de Vida em Crianças: Perspectivas no Brasil e no mundo. J. Pediatr. (Rio J.). 2007;83(2 Supl):S109-16. https://doi.org/10.1590/ $\underline{\mathrm{S} 0021-75572007000300013}$ 
9. Minosso JSM, Martins MMFPS, Oliveira MAC. Adaptação transcultural do Bonn Palliative Care Knowledge Test: um instrumento para avaliar conhecimentos e autoeficácia. Revista de Enfermagem Referência. 2017;4(13):31-42. https://doi. org/10.12707/RIV16076

10. Kirby E, Broom A, Good P. The role and significance of nurses in managing transitions to palliative care: a qualitative study. BMJ Open. 2014;4(9):e006026. https://doi.org/10.1136/ bmjopen-2014-006026

11. Moser AD, Scharan K. O Olhar Biopsicossocial na Fisioterapia: ferramentas disponíveis para sua operacionalização. Fisioter. Mov. 2018;31:e003136. http://dx.doi.org/10.1590/1980-5918.031. ed01

12. Oliveira LCM, Teixeira VL, Tavares GR. Cuidados paliativos no CTI de um Hospital Universitário: a percepção dos profissionais de saúde. Revista Interdisciplinar Ciências Médicas [Internet]. 2019;3(2):36-41. Disponível em: http://revista.fcmmg.br/ojs/index. php/ricm/article/view/236/75

13. Portaria 4.279, de 30 de dezembro de 2010 (Brasil). Estabelece diretrizes para a organização da Rede de atenção à saúde no âmbito do Sistema Único de Saúde [Internet]. Diário Oficial da União; 2010. 31 dez. 2010. Disponível em: http://bvsms.saude.gov. br/bvs/saudelegis/gm/2010/prt4279_30_12_2010.html

14. Azevedo C, Rates CMP, Pessalacia JDR, Mata LRF. Perspectivas para os cuidados paliativos na atenção primária à saúde: estudo descritivo. Online braz j nurs [Internet]. 2016;15(4):683-93. Disponível em: https://docs.bvsalud.org/ biblioref/2019/03/967511/objn-2016.pdf

15. Furtado CR, Gehlen MH, Ventura J, Paula SF, Pereira $A D$, Ferreira $C L$, et al. $A$ bioética no cotidiano hospitalar e o desenvolvimento dos cuidados paliativos pela equipe de enfermagem. Disciplinarum Scientia [Internet]. 2018;19(2):245-53. Disponível em: https://periodicos.ufn.edu.br/index.php/disciplinarums/article/ view/2509
16. Valadares MTM, Mota JAC, Oliveira BM. Cuidados Paliativos em Pediatria: uma revisão. Rev. Bioét. 2013;21(3):486-93. https://doi. org/10.1590/S1983-80422013000300013

17. Castro A, Testoni I, Zamperini A, Ronconi L, Galantin LP, Caraceni A. The implicit soul: Factors between the representation of death and dehumanization of patients. Health Psychol Open. 2019;6(1):2055102919854666. https://doi. org/10.1177/2055102919854666

18. Spineli VMCD. Conhecimento e autoeficácia em Cuidados Paliativos de enfermeiros da atenção Primária à Saúde [tese] [Internet]. São Paulo: Universidade de São Paulo; 2019. Disponível em: https://www.teses.usp.br/teses/disponiveis/7/7143/tde11122019-165525/pt-br.php

19. Brito FM, Coutinho MJF, Andrade CG, Costa SFG, Costa ICP, Santos KFO. Cuidados paliativos e comunicação: estudo com profissionais de saúde do serviço de atenção domiciliar. R. pesq. cuid. fundam. online. 2017;9(1):215-21. https://doi. org/10.9789/2175-5361.2017.v9i1.215-221

20. Bastos BR, Fonseca ACG, Pereira AKS, Silva LCS. Formação dos profissionais de saúde na comunicação de más notícias em cuidados paliativos oncológicos. Revista Brasileira de Cancerologia [Internet]. 2016;62(3):263-6. Disponível em: https://rbc.inca.gov.br/site/arquivos/n_62/v03/pdf/10-artigoopiniao-formacao-dos-profissionais-de-saude-na-comunicacaode-mas-noticias-em-cuidados-paliativos-oncologicos.pdf

21. Pfister D, Müller M, Müller S, Kern M, Rolke R, Radbruch L. Validierung des Bonner Palliativwissenstests (BPW) [Validation of the Bonn test for knowledge in palliative care (BPW)]. Schmerz. 2011;25(6):643-53. https://doi.org/10.1007/s00482-011-1111-7

22. Pfister D, Markett S, Müller M, Müller S, Grützner F, Rolke R, et al. German nursing home professionals' knowledge and specific self-efficacy related to palliative care. J Palliat Med. 2013;16(7):7948. https://doi.org/10.1089/jpm.2012.0586 